\title{
Dynamically remodeled hepatic extracellular matrix predicts prognosis of early-stage cirrhosis
}

\author{
Yuexin $\mathrm{Wu}^{1,2}$, Yuyan Cao ${ }^{1,2}$, Keren $\mathrm{Xu}^{2,3}$, Yue Zhu ${ }^{1,2}$, Yuemei Qiao', Yanjun Wu', Jianfeng Chen ${ }^{1,4}$, Chen Li ${ }^{3,5}$, \\ Rong Zeng $\mathbb{1}^{3,6,7}$ and Gaoxiang Ge (1)
}

\begin{abstract}
Liver cirrhosis remains major health problem. Despite the progress in diagnosis of asymptomatic early-stage cirrhosis, prognostic biomarkers are needed to identify cirrhotic patients at high risk developing advanced stage disease. Liver cirrhosis is the result of deregulated wound healing and is featured by aberrant extracellular matrix (ECM) remodeling. However, it is not comprehensively understood how ECM is dynamically remodeled in the progressive development of liver cirrhosis. It is yet unknown whether ECM signature is of predictive value in determining prognosis of early-stage liver cirrhosis. In this study, we systematically analyzed proteomics of decellularized hepatic matrix and identified four unique clusters of ECM proteins at tissue damage/inflammation, transitional ECM remodeling or fibrogenesis stage in carbon tetrachloride-induced liver fibrosis. In particular, basement membrane (BM) was heavily deposited at the fibrogenesis stage. BM component minor type IV collagen a 5 chain expression was increased in activated hepatic stellate cells. Knockout of minor type IV collagen a5 chain ameliorated liver fibrosis by hampering hepatic stellate cell activation and promoting hepatocyte proliferation. ECM signatures were differentially enriched in the biopsies of good and poor prognosis early-stage liver cirrhosis patients. Clusters of ECM proteins responsible for homeostatic remodeling and tissue fibrogenesis, as well as basement membrane signature were significantly associated with disease progression and patient survival. In particular, a 14-gene signature consisting of basement membrane proteins is potent in predicting disease progression and patient survival. Thus, the ECM signatures are potential prognostic biomarkers to identify cirrhotic patients at high risk developing advanced stage disease.
\end{abstract}

Liver cirrhosis remains major health problem ${ }^{1,2}$. Nearly all chronic liver diseases eventually lead to liver fibrosis and cirrhosis $^{1,2}$. Fibrotic liver diseases predispose to liver failure, portal hypertension, and are associated with increased risk of liver cancer ${ }^{1,2}$. Serum biomarkers and liver stiffness measurement are becoming increasingly useful in liver cirrhosis diagnosis, in particular

\footnotetext{
Correspondence: Chen Li (cli@sibs.ac.cn) or Rong Zeng (zr@sibcb.ac.cn) or Gaoxiang Ge (gxge@sibcb.ac.cn)

${ }^{1}$ State Key Laboratory of Cell Biology, Shanghai Institute of Biochemistry and Cell Biology, Center for Excellence in Molecular Cell Science, Chinese Academy of Sciences, 200031 Shanghai, China

2University of Chinese Academy of Sciences, 100049 Beijing, China

Full list of author information is available at the end of the article

These authors contributed equally: Yuexin Wu, Yuyan Cao

Edited by L. Sun
}

asymptomatic early-stage cirrhosis ${ }^{3}$. However, prognostic biomarkers are needed to identify cirrhotic patients at high risk developing advanced stage disease. Child-Pugh score, determined by scoring five clinical measures, including total bilirubin, serum albumin, prothrombin time, presence of ascites and hepatic encephalopathy, is used to assess the prognosis of chronic liver disease, in particular cirrhosis ${ }^{4}$. Child-Pugh Class A liver cirrhosis patients have the least severe liver disease and the highest survival rate, compared to the Class B (moderately severe liver disease) or Class $\mathrm{C}$ (most severe liver disease) patients $^{5,6}$. A 186-gene signature has been shown to predict clinical outcomes of patients with hepatitis C-related Class A early-stage cirrhosis ${ }^{5}$. Despite these progresses, there is still urgent need for robust and

\section{(c) The Author(s) 2021}

(c) Open Access This article is licensed under a Creative Commons Attribution 4.0 International License, which permits use, sharing, adaptation, distribution and reproduction cc) in any medium or format, as long as you give appropriate credit to the original author(s) and the source, provide a link to the Creative Commons license, and indicate if changes were made. The images or other third party material in this article are included in the article's Creative Commons license, unless indicated otherwise in a credit line to the material. If material is not included in the article's Creative Commons license and your intended use is not permitted by statutory regulation or exceeds the permitted use, you will need to obtain permission directly from the copyright holder. To view a copy of this license, visit http://creativecommons.org/licenses/by/4.0/. 
sensitive prognostic biomarkers for patients with liver cirrhosis, especially early-stage liver cirrhosis.

Liver fibrosis is part of the general wound healing response to many causes of chronic injury ${ }^{7,8}$. Chronic injury causes sustained inflammation, myofibroblast activation and aberrant extracellular matrix (ECM) deposition. Bioinformatic analysis of proteins containing characteristic domains commonly found in ECM proteins, as well as proteomic analyses of proteins in purified ECM have identified $\sim 300$ core ECM proteins, including $\sim 200$ glycoproteins, 43 collagens, and 36 proteoglycans (http:// matrisomeproject.mit.edu $)^{9,10}$. In addition, there are large numbers of ECM-associated proteins, including ECMaffiliated proteins, which share domain similarity with core ECM proteins or are known to be associated with ECM proteins, ECM regulators that are responsible for regulating ECM remodeling, and secreted factors bound to core ECM proteins ${ }^{9,10}$. Core ECM proteins and ECM-associated proteins are collectively known as matrisome ${ }^{9,10}$. Core ECM proteins and ECM-associated proteins cooperatively assemble and remodel ECM and regulate cellular functions through cell surface receptors. Compositions of matrisome are qualitatively and quantitatively different in normal and diseased tissues $^{10-18}$. Such ECM changes are highly dynamic during wound healing and tissue fibrosis. In skin wound healing, types XII and XIV collagens and tenascin C are found throughout skin wound healing, while latent TGF- $\beta$ binding protein- 4 is detected in the later phase of wound repair ${ }^{11}$. Fibronectin assembles into fibrillar structures that provide scaffold for collagen fibril assembly. Deposition of fibronectin is an early event in both wound healing and tissue fibrosis ${ }^{19,20}$. Blocking fibronectin deposition decreases collagen accumulation and improves liver function during liver fibrogenesis ${ }^{21}$. Ethanol and/or acute lipopolysaccharide challenge induce transitional ECM remodeling and dramatically increase the number of hepatic ECM proteins before fibrotic changes are evident in the liver ${ }^{12}$.

We thus hypothesize that dynamically remodeled hepatic matrix may possess prognostic value for liver fibrosis and cirrhosis. Here, we used enriched hepatic matrix and proteomic approach to study the dynamic changes of the ECM during the progression of experimental liver fibrosis. Unique matrisomal proteins were identified for tissue damage/inflammation, transitional ECM remodeling and fibrogenesis. Such matrisomal protein signatures, including basement membrane signature, are predictive of Child-Pugh Class A earlystage cirrhosis progression and patient survival. A 14-gene signature, present in both cluster 4 and basement membrane signatures, predicts progression and patient survival of Child-Pugh Class A early-stage cirrhosis.

\section{Results}

Global changes of the hepatic matrisomal proteins in liver fibrosis

Repeated carbon tetrachloride $\left(\mathrm{CCl}_{4}\right)$ treatment leads to iterative wound healing, resulting in deregulated ECM deposition and progressive development of liver fibro$\mathrm{sis}^{22,23}$. At 1-day after $\mathrm{CCl}_{4}$ treatment, there were large areas of hepatocyte death and inflammation, as well as large number of proliferating cells (Fig. 1A and S1). At 1week after $\mathrm{CCl}_{4}$ treatment, hepatic necrosis was not evident, and the inflammation and cell proliferation ceased (Fig. 1A and S1). Weak collagen deposition was observed after 1-week $\mathrm{CCl}_{4}$ treatment (Fig. 1A). 4-week repeated $\mathrm{CCl}_{4}$ treatment resulted in extensive collagen deposition and $\alpha$-smooth muscle actin ( $\alpha \mathrm{SMA})$-positive myofibroblast accumulation (Fig. 1A and S1). Deposition of fibronectin is an early event in tissue fibrosis that provides scaffold for collagen fibril assembly ${ }^{19,20}$. Fibronectin was deposited as early as 1-day after $\mathrm{CCl}_{4}$ treatment and heavily deposited in the fibrotic livers after 4-week $\mathrm{CCl}_{4}$ treatment (Fig. 1A). 1-day, 1-week and 4-week $\mathrm{CCl}_{4}$ treatment thus represented stages of tissue damage/inflammation, transitional ECM remodeling and fibrogenesis.

During the development of liver fibrosis, ECM undergoes dynamic remodeling. Decellularization by non-ionic detergents removes soluble intracellular and extracellular proteins, while preserves insoluble ECM scaffold and proteins tightly bound to the ECM scaffold ${ }^{24}$. Hepatic matrices were enriched and subjected to in-depth proteomic analyses. Proline and lysine residues are heavily hydroxylated in collagens and other ECM proteins ${ }^{10,25,26}$. To estimate the abundance of matrisomal proteins more accurately, both proline and lysine hydroxylation was included in database search ${ }^{13}$. A total of 152 matrisomal proteins were identified, in which 81 matrisomal proteins were identified in all 4 groups (Figs. 1B, C and Table S1). Each group yielded a distinct ECM protein profile (Fig. $1 \mathrm{C}$ and Table S1). In particular, 7 (LAMA1, LGALSL, MBL, CTSG, MASP1, SERPINB1A, SERPINB9C) and 12 (MFGE8, ASPN, CST3, F10, HYAL1, MMP19, P4HA2, SERPINA1C, SERPINA1D, SERPIND1, SERPINF1, NGF) matrisomal proteins were only identified in the 1-day and 1-week treatment groups, respectively (Fig. $1 \mathrm{C}$ and Table S1). Additional 15 matrisomal proteins were identified in both 1-day and 1-week treatment groups (CRELD2, IGFBP1, TSKU, PRG4, CSTB, F12, HABP2, SERPINA1B, SERPINA1E, SERPINA3N, SERPINA7, SERPINF2, ANGPTL4, CRLF3, S100A8), but not in the control and 4-week groups (Fig. $1 \mathrm{C}$ and Table S1), suggesting the transitional nature at these two time points. COL15A1 and P4HA1 were only identified in the 4-week treatment group (Fig. 1C and Table S1). Gene Ontology (GO) analysis revealed that these matrisomal proteins are involved 


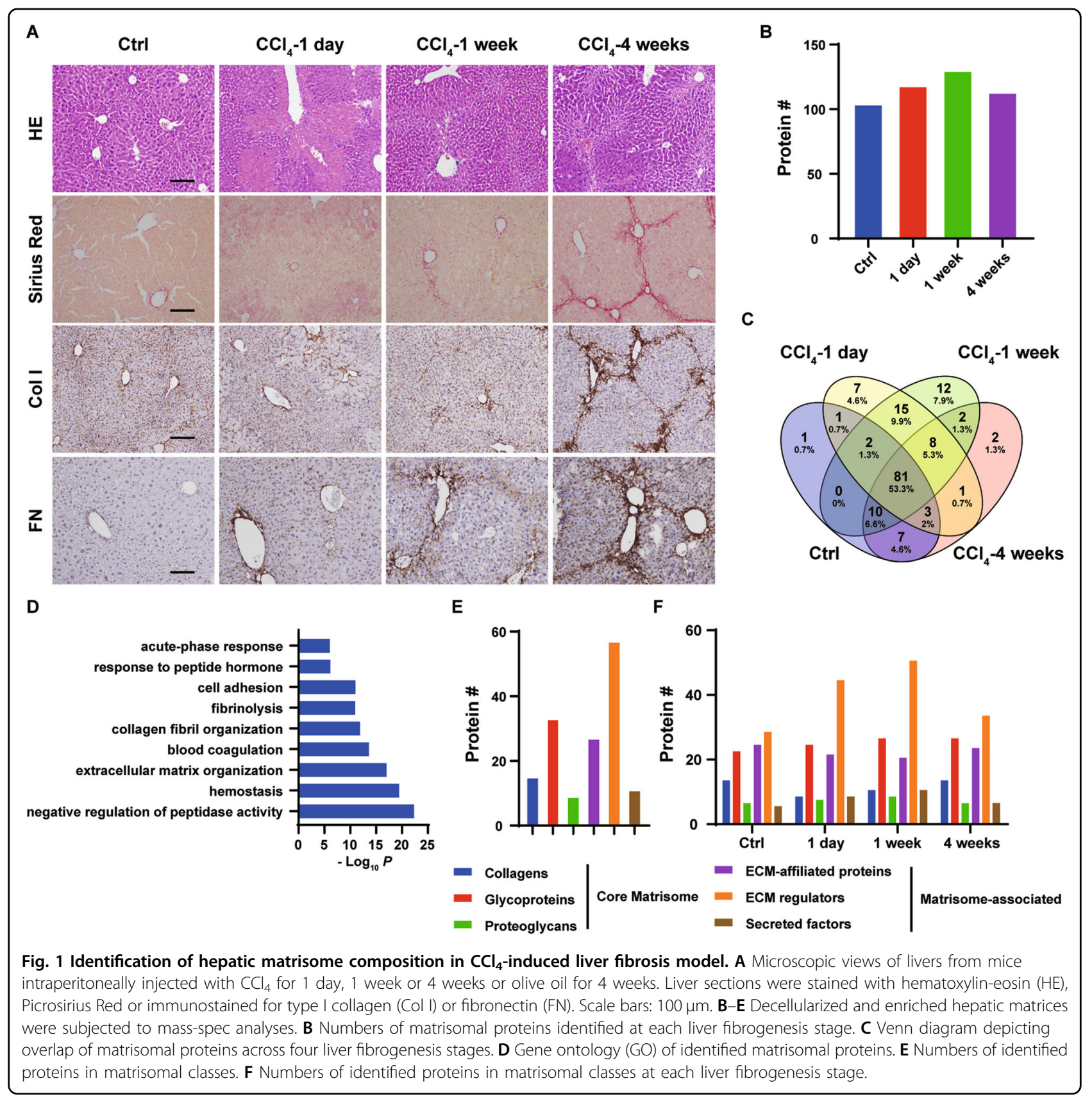

in negative regulation of peptidase activity and ECM organization (Fig. 1D).

\section{Differential changes of the matrisome subgroups in liver fibrosis}

The matrisome comprises structural ECM proteins, termed as core ECM, as well as ECM-associated proteins ${ }^{10}$. A total of 57 core ECM proteins (15 collagens, 33 glycoproteins and 9 proteoglycans) and 95 ECMassociated proteins (27 ECM-affiliated proteins, 57 ECM regulators and 11 secreted factors) were identified (Fig. 1E). The numbers of identified glycoproteins, proteoglycans, ECM-affiliated proteins and secreted factors were comparable among different groups (Fig. 1F, Table S1). However, the numbers of identified collagens and ECM regulators drastically changed after 1-day and 1week $\mathrm{CCl}_{4}$ treatment (Fig. 1F, Table S1). Less collagen proteins were identified (9 and 11 vs. 14 in control and 4week groups), while more ECM regulators were detected (45 and 51 vs. 29 and 34 in control and 4-week groups) after 1-day and 1-week $\mathrm{CCl}_{4}$ treatment (Fig. 1F, Table S1). Gene set enrichment analysis (GSEA) ${ }^{27}$ revealed dynamic changes of core matrisome and matrisome-associated proteins during $\mathrm{CCl}_{4}$-induced liver fibrosis (Fig. S2). 
Compared to the control group, significantly downregulated core matrisome protein ${ }^{10}$ expression and upregulated matrisome-associated protein ${ }^{10}$ expression was detected after 1-day $\mathrm{CCl}_{4}$ treatment (Fig. S2). Consistent to the prominent liver fibrosis, core matrisome was significantly enriched, whereas matrisome-associated protein expression was significantly decreased after 4-week $\mathrm{CCl}_{4}$ treatment (Fig. S2). The subgroups of matrisomal proteins were differentially regulated during $\mathrm{CCl}_{4}$-induced liver fibrosis. Both collagens ${ }^{10}$ and proteoglycans ${ }^{10}$ were quantitatively downregulated after 1-day treatment, compared to the control group (Fig. S3A and S3C). Collagen expression was only restored after 4-week, but not 1-week $\mathrm{CCl}_{4}$ treatment (Fig. S3A). Unlike the collagens, expression of proteoglycans ${ }^{10}$ was partially restored in 1-week group that was further increased in 4-week group (Fig. S3C). Expression of ECM regulators ${ }^{10}$ was significantly upregulated in 1-day group, compared to the control group that was further upregulated after 1-week treatment (Fig. S3E). Expression of ECM regulators ${ }^{10}$ was sharply decreased after 4-week treatment (Fig. S3E). Expression of glycoproteins, ECM-affiliated protein and secreted factors ${ }^{10}$ only moderately changed during $\mathrm{CCl}_{4}$-induced liver fibrosis (Fig. S3B, S3D and S3F).

\section{Dynamic changes of the matrisome in liver fibrosis}

Matrisomal proteins changed in relative abundance in response to $\mathrm{CCl}_{4}$ treatment. Clustering analysis of matrisomal protein quantitation identified four clusters (Fig. 2A-C and Table S2). Cluster 1 represented matrisomal proteins upregulated at 1-day after $\mathrm{CCl}_{4}$ treatment, containing mainly glycoproteins, ECM-affiliated proteins, and ECM regulators. Cluster 2 represented matrisomal proteins upregulated at 1-week after $\mathrm{CCl}_{4}$ treatment, containing mainly ECM regulators and ECM-affiliated proteins. Cluster 3 represented matrisomal proteins upregulated at 1-day and 1-week after $\mathrm{CCl}_{4}$ treatment, containing mainly ECM regulators and glycoproteins. Cluster 4 represented matrisomal proteins decreased at 1day or 1-week, but restored at 4-week after $\mathrm{CCl}_{4}$ treatment, containing mainly collagens, glycoproteins, and ECM regulators. Consistent to the histological changes of acute tissue damage/inflammation at 1-day, transitional ECM remodeling at 1-week, and fibrogenesis at 4-week after $\mathrm{CCl}_{4}$ treatment (Fig. 1A), GO analysis revealed each cluster was associated with distinct biological processes (Fig. 2D). Cluster 1 contained proteins involved in the regulation of tissue damage and immune responses. Cluster 2 and 3 contained proteins involved in the negative regulation of peptidase activity and ECM remodeling. Cluster 4 contained proteins that are involved in the regulation of collagen fibril and ECM organization. Glycoproteins fibrinogen chains (FGA, FGB, FGG) and secreted factors S100A8/S100A ${ }^{28}$ responsible for blood clotting and active inflammatory response were highly expressed at 1-day, but expression of these glycoproteins sharply decreased at 1-week after $\mathrm{CCl}_{4}$ treatment (Fig. S4). Expression of laminin and periostin, glycoproteins required for liver fibrosis development ${ }^{29}$, decreased at 1day and 1-week, but restored at 4-week after $\mathrm{CCl}_{4}$ treatment (Fig. S4). Type V collagen is a low abundance fibrillar collagen incorporated into type I collagen fibrils to regulate the size and shape of the type I collagen fibrils ${ }^{30}$. Similar to that of type I collagen, expression of type $\mathrm{V}$ collagen decreased at 1-day, but increased at 4week after $\mathrm{CCl}_{4}$ treatment (Fig. S4).

Cirrhosis represents the terminal stage of chronic fibrotic liver diseases. In addition to clinical prognostic variables, e. g., platelet count and serum bilirubin levels, 186-gene signature was used to predict outcomes of patients with hepatitis C-related Child-Pugh Class A early-stage cirrhosis, and classify the patients as having poor and good prognosis of disease progression to Class B or $\mathrm{C}$ advanced stage disease and patient death ${ }^{5}$. Expression of cluster-1, cluster-2, or cluster-3 matrisomal proteins were significantly enriched in the liver biopsies from the Class A early-stage cirrhosis patients least likely developing Class B or Class $\mathrm{C}$ advanced stage disease (good prognosis group), compared to that from the patients at high risk developing Class B or Class C advanced stage disease (poor prognosis group) (Fig. 2E and $\mathrm{S} 5 \mathrm{~A}$ ). In contrast, cluster-4 matrisomal proteins were enriched in the poor prognosis group (Fig. 2E and S5A). The differential enrichment of each cluster indicated that dynamically remodeled ECM is actively involved in disease progression. We next sought to determine whether the clinical outcomes of the cirrhotic patients could be predicted by expression of the ECM clusters in the ChildPugh Class A early-stage liver cirrhosis samples ${ }^{5}$. Cox analyses indicated that clusters- 3 and -4 were predictive for the progression of Child-Pugh class and patient survival (Tables S3). To avoid potential heterogeneity among the samples, we randomly split 216 samples into two data sets with similar size (107 and 109 cases) and similar clinicopathologic characteristics (Table S4). Patients with low cluster-3 and high cluster-4 matrisomal protein expression had significantly shorter disease progressionfree survival time than the rest of the patients (low cluster-3/low cluster-4, high cluster-3/low cluster-4, or high cluster-3/high cluster-4 matrisomal protein expression) in both Cohort-1 and Cohort-2 (Cohort-1: HR: 3.29 (1.44-7.54), $P=0.0047$; Cohort-2: HR: 6.06 (2.59-14.19), $P<0.0001$ ) (Fig. 2F). Ten-year rates of Child-Pugh class progression were $44 \%$ vs. $12 \%$ (Cohort-1) and $65 \%$ vs. $27 \%$ (Cohort-2) for patients with or without low Cluster 3/high cluster 4 matrisomal protein expression pattern (Fig. 2F). Patients with low cluster-3/high cluster-4 matrisomal protein expression had significantly shorter overall 
A

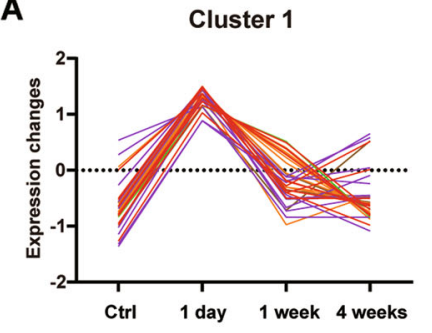

Cluster 3

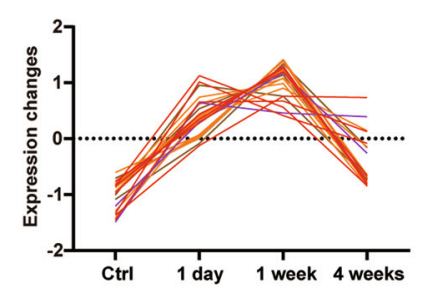

B

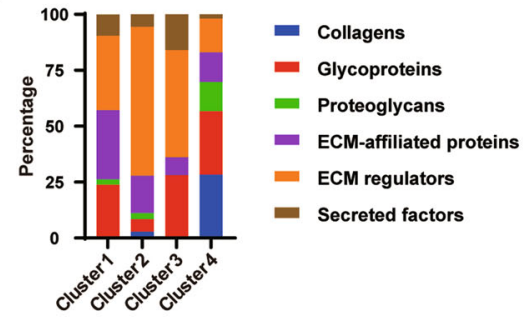

D

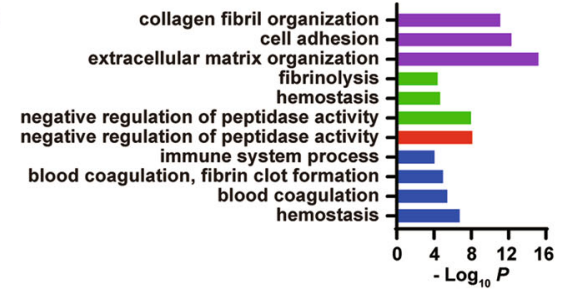

E

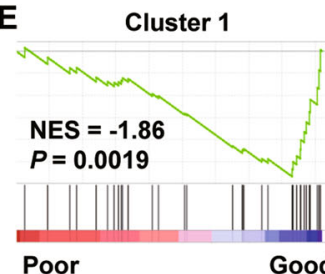

Poor

$\mathbf{F}$

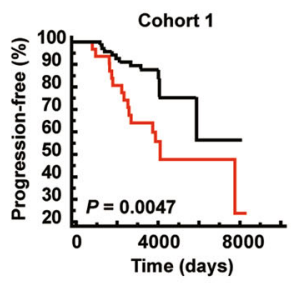

Good Poor

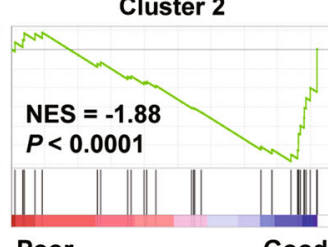

Good Poor

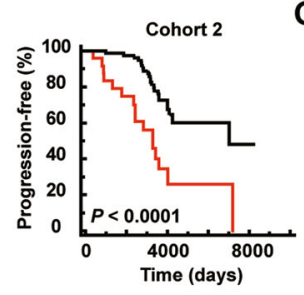

- Cluster 3 low, Cluster 4 high

-_ Non-Cluster 3 low, Cluster 4 high
Cluster 2
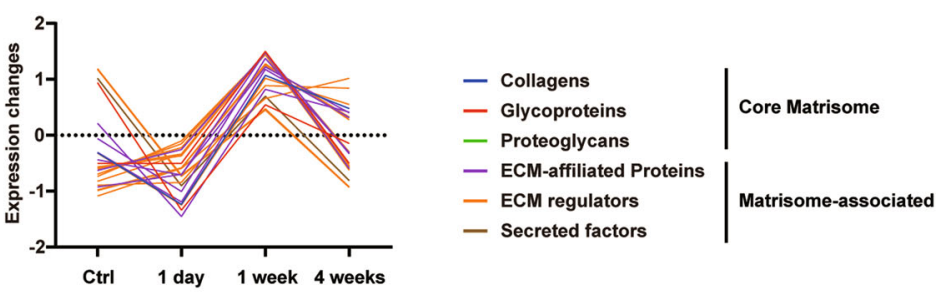

Cluster 4

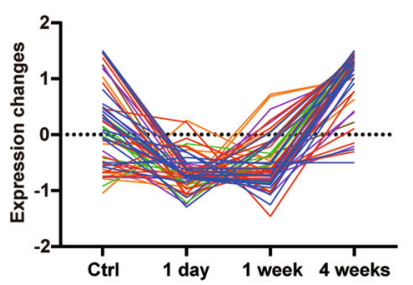

C

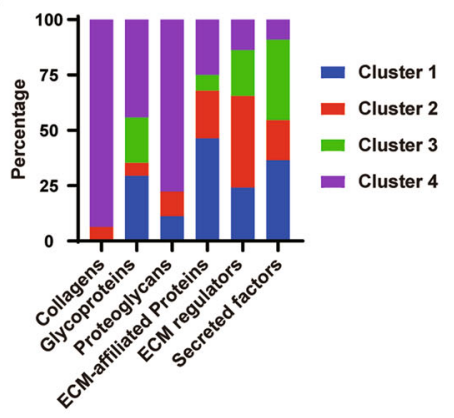

- Cluster 1

- Cluster 2

- Cluster 3

- Cluster 4 
(see figure on previous page)

Fig. 2 Hepatic proteins in matrisomal classes are dynamically remodeled in $\mathrm{CCl}_{4}$-induced liver fibrosis model, and predict disease progression and survival of early-stage liver cirrhosis patients. A Clustered profiles of protein abundances for identified matrisomal proteins. B Distribution of matrisomal classes in each cluster. C Distribution of clusters in each matrisomal class. D Gene ontology (GO) of each cluster. E GSEA analyses comparing enrichment of ECM clusters between good prognosis and poor prognosis Child-Pugh Class A early-stage liver cirrhosis biopsies. F, G Probabilities of disease progression from Child-Pugh Class A to Class B or C (F) and survival $(\mathbf{G})$ of early-stage cirrhosis patients according to the expression levels of the clusters-3 and cluster-4 ECM signatures.

survival time than the patients without low cluster-3/high cluster-4 matrisomal protein expression pattern (Cohort1: HR: 2.46 (1.13-5.39), $P=0.024$; Cohort-2: HR: 8.26 (3.38-20.20), $P<0.0001$ ) (Fig. $2 \mathrm{G})$. Ten-year survival rates were $64 \%$ vs. $84 \%$ (Cohort-1) and $38 \%$ vs. $80 \%$ (Cohort- 2 ) for patients with or without low cluster-3/high cluster-4 matrisomal protein expression pattern (Fig. 2G).

\section{Basement membrane proteins are prognostic for early- stage liver cirrhosis}

BMs are specialized ECMs underneath epithelial cells, endothelial cells, and smooth muscle cells ${ }^{31,32}$. Type IV collagens, laminins, perlecan, nidogens, type XVIII collagen and type XV collagen are the core BM components ${ }^{10,33}$. Consistent to damaged tissue architecture, core $\mathrm{BM}$ components ${ }^{10}$ were downregulated after 1-day $\mathrm{CCl}_{4}$ treatment (Fig. 3A). BMs were partially restored after 1week and fully restored after 4-week $\mathrm{CCl}_{4}$ treatment (Fig. 3A). BMs are highly upregulated in liver fibrosis and cirrhosis $^{34,35}$. Core BM components were enriched in the poor prognosis Child-Pugh Class A liver cirrhosis patient group (Fig. 3B and S5B). High core BM protein expression correlated to shorter disease progression-free (Cohort-1: HR: 2.97 (1.38-6.42), $P=0.0056$; Cohort-2: HR: 5.75 (2.94-11.24), $P<0.0001$ ) and overall survival (Cohort-1: HR: 3.57 (1.71-7.44), $P=0.0007$; Cohort-2: HR: 3.07 (1.53-6.17), $P=0.0016$ ) (Figs. 3C, D). Ten-year ChildPugh class progression rates were $32 \%$ vs. $11 \%$ (Cohort- 1 ) and $67 \%$ vs. $13 \%$ (Cohort-2) for patients with high or low BM signature (Fig. 3C). Ten-year survival rates were $63 \%$ vs. $90 \%$ (Cohort- 1 ) and $43 \%$ vs. $85 \%$ (Cohort-2) for patients with high or low BM signature (Fig. 3D). Clinical variables, e. g. bilirubin level and platelet count, are used to assess the prognosis of liver cirrhosis ${ }^{4}$. Multivariable Cox analyses showed that the basement membrane signature remained significant for the association with progression of Child-Pugh class (HR: 3.19 (1.87-5.42), $P<$ 0.0001 ) and patient death (HR: 2.57 (1.54-4.30), $P=$ 0.0003) (Table S5). The presence of both high basement membrane signature and high bilirubin level increased the hazard ratio for progression of Child-Pugh class (HR: 10.71 (4.47-25.64), $P<0.0001)$ and patient death (HR: 6.79 (3.29-14.01), $P<0.0001$ ) (Table S6), suggesting that basement membrane signature and bilirubin level is complementary in determining patient prognosis. Indeed, patients with both variables high had the shortest disease progression-free survival $(P<0.0001)$ and overall survival $(P<0.0001)$ (Fig. S6A and S6B). Ten-year Child-Pugh class progression rates were 62 and $4 \%$ for patients with high BM signature/high bilirubin level and low BM signature/low bilirubin level, respectively (Fig. S6A). Ten-year survival rates were 40 and $90 \%$ for patients with high BM signature/high bilirubin level and low BM signature/low bilirubin level, respectively (Fig. S6B).

Cluster-4 and basement membrane signatures share 14 common proteins, including $\alpha$ chains of type IV, VI, XV and XVIII collagens, laminins, agrin, nidogen and HSPG2 (Fig. 3E, Table S8). Such 14-gene signature is highly enriched in the poor prognosis group of ChildPugh Class A liver cirrhosis patient samples (Fig. 3F). Univariable Cox analyses indicated that the 14-gene were associated with disease progression (HR: 3.11, $P<$ 0.0001 ) and patient survival (HR: 2.14, $P=0.0022$ ) (Table S3). High 14-gene signature expression correlated to shorter disease progression-free (Cohort-1: HR: 3.71 (1.69-8.14), $P=0.0011$; Cohort-2: HR: 1.57 (1.57-5.82), $P=0.001)$ and overall survival (Cohort-1: HR: 2.79 (1.32-5.88), $P=0.0071$; Cohort-2: HR: 1.87 (0.94-3.71), $P=0.074)$ (Figs. 3G, H). Ten-year Child-Pugh class progression rates were $32 \%$ vs. $12 \%$ (Cohort-1) and $58 \%$ vs. $19 \%$ (Cohort-2) for patients with high or low 14-gene signature (Fig. 3C). Ten-year survival rates were 65\% vs. $87 \%$ (Cohort- 1 ) and $53 \%$ vs. $80 \%$ (Cohort-2) for patients with high or low 14-gene signature (Fig. 3D). 14-gene signature was significantly association with progression of Child-Pugh class (HR: 2.83 (1.70-4.70), $P<0.0001)$ and patient death (HR: $2.07(1.26-3.39), P=0.0042)$ in multivariable Cox analyses (Table S5). The presence of both 14-gene signature and high bilirubin level increased the risk of disease progression (HR: 6.12 (2.75-13.63), $P$ $<0.0001$ ) and patient death (HR: 4.01 (1.97-8.20), $P=0.0001$ ) (Table S6). Patients with high 14-gene signature and high bilirubin level had the shortest progression-free survival $(P<0.0001)$ and overall survival $(P<0.0001)$ (Fig. S6C and S6D). Ten-year Child-Pugh class progression rates were 62 and $6 \%$ for patients with high 14-gene signature/high bilirubin level and low 14-gene signature/low bilirubin level, respectively (Fig. S6C). Ten-year survival rates were 43 and $88 \%$ for patients with high 14-gene signature/high bilirubin level and low 14-gene signature/low bilirubin level, respectively (Fig. S6D). 


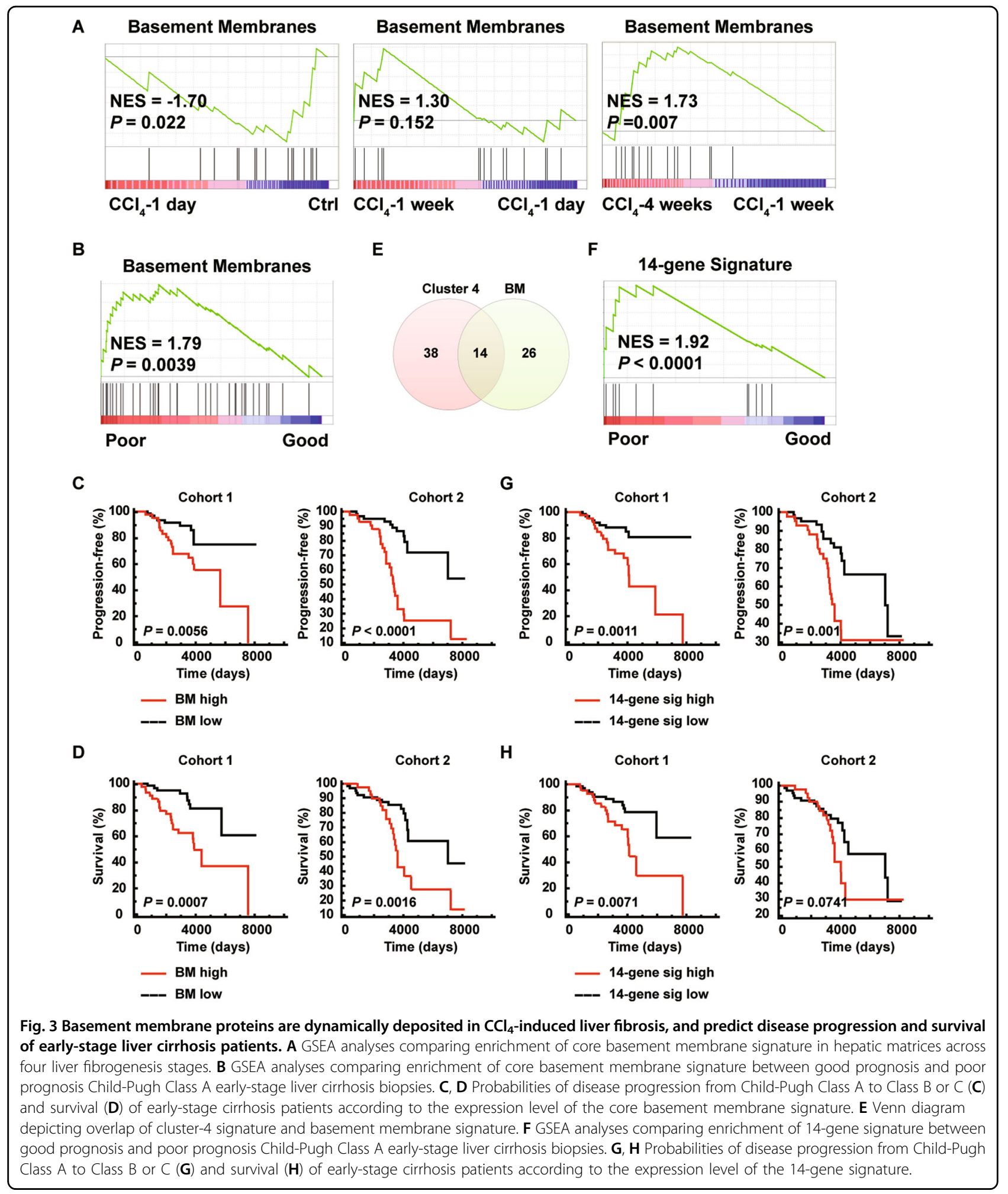

Minor type IV collagens are prominent for the development of liver fibrosis

Type IV collagens, the core components of BMs essential for the maintenance of BM structure ${ }^{36,37}$, were expressed in the liver and highly upregulated after 4-week $\mathrm{CCl}_{4}$ treatment (Figs. 4A, B), consistent to the findings that type IV collagens are accumulated in human liver fibrosis and cirrhosis ${ }^{34,35}$. There are 6 highly homologous 


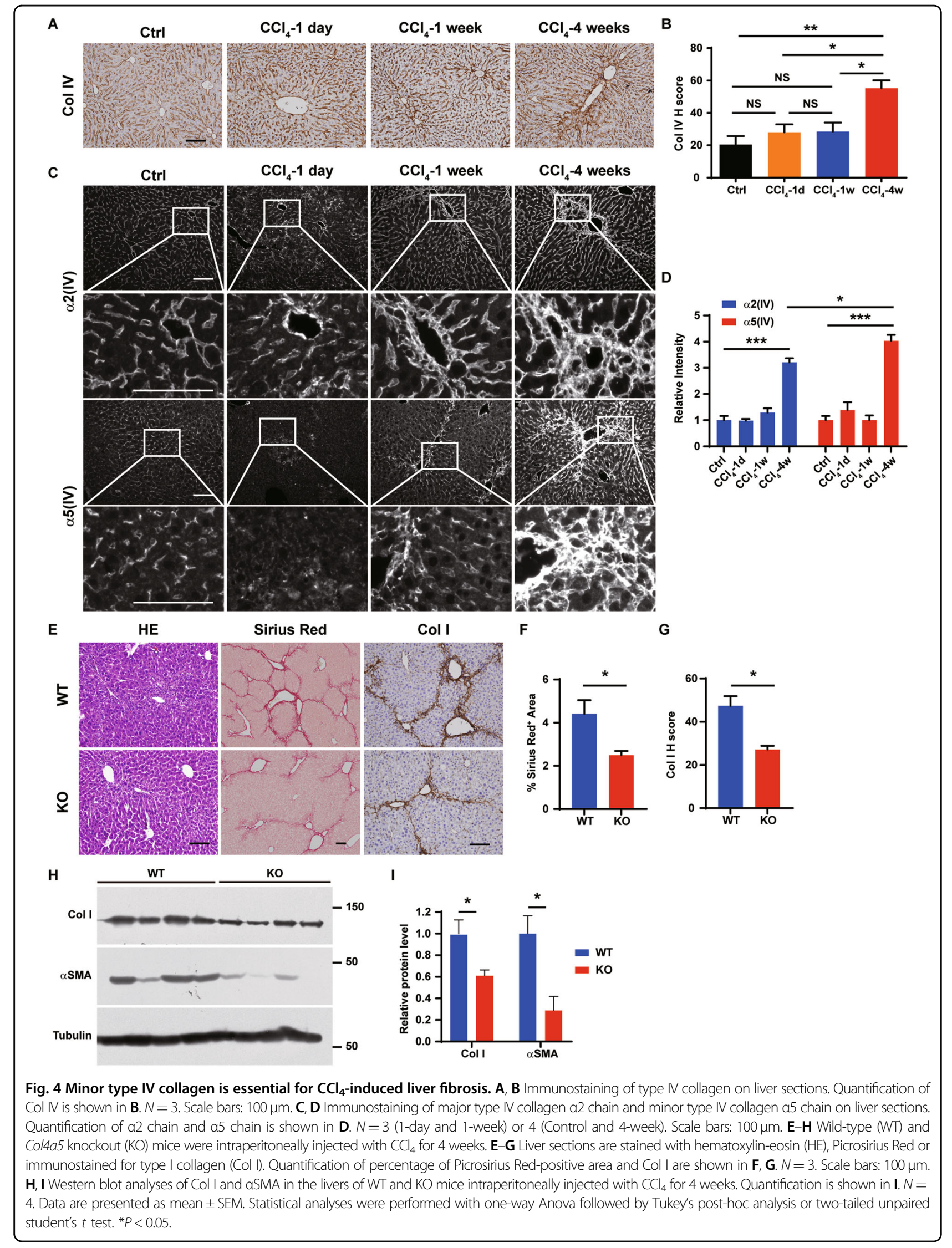


type IV collagen $\alpha$ chains $(\alpha 1(\mathrm{IV})-\alpha 6(\mathrm{IV}))^{36,37}$. Type IV collagen $\alpha$ chains form $\alpha 1 \alpha 1 \alpha 2(\mathrm{IV}), \alpha 3 \alpha 4 \alpha 5$ (IV), and $\alpha 5 \alpha 5 \alpha 6$ (IV) heterotrimers, in which $\alpha 1 \alpha 1 \alpha 2$ (IV), known as major type IV collagen, is broadly and abundantly expressed and $\alpha 3 \alpha 4 \alpha 5$ (IV) and $\alpha 5 \alpha 5 \alpha 6$ (IV), known as minor type IV collagens, are expressed at low abundance $^{36,37}$. Peptides of type IV collagens identified in the proteomic analyses were conserved between $\alpha 1(\mathrm{IV})$ and $\alpha 5(I V)$ chains (Table S7). Both major type IV collagen $\alpha 2$ (IV) chain and minor type IV collagen $\alpha 5$ (IV) chain were localized to the Disse space in the liver (Fig. 4C). Major type IV collagen was unchanged after 1-day and 1-week $\mathrm{CCl}_{4}$ treatment, but was accumulated (3.2-fold compared to Oil control) at the fibrotic lesions after 4-week $\mathrm{CCl}_{4}$ treatment (Figs. $4 \mathrm{C}, \mathrm{D}$ and $\mathrm{S7}$ ). $\alpha 5(\mathrm{IV})$ was more substantially upregulated (4-fold compared to Oil control) than $\alpha 2(\mathrm{IV})$ after 4-week $\mathrm{CCl}_{4}$ treatment (Figs. $4 \mathrm{C}, \mathrm{D}$ and S7). Despite the total signal intensity of $\alpha 5$ (IV) was not changed after 1-day $\mathrm{CCl}_{4}$ treatment, $\alpha 5$ (IV) was only detected as fragmented dot-like structure, unlike the continuous structures observed in the control livers or after 1-week or 4-week $\mathrm{CCl}_{4}$ treatment, indicating disrupted $\alpha 5$ (IV) structure after 1 -day $\mathrm{CCl}_{4}$ treatment (Fig. $4 C)$. Unlike $\alpha 5(\mathrm{IV}), \alpha 2(\mathrm{IV})$ was present as normal continuous structures at all stages during $\mathrm{CCl}_{4}$-induced liver fibrosis (Fig. 4C). The dynamic changes of minor type IV collagen expression and structure in $\mathrm{CCl}_{4}$-induced liver fibrosis indicated that minor type IV collagens may play prominent regulatory roles in hepatic fibrogenesis.

To investigate the functions of minor type IV collagens in liver fibrosis, mice deficient of $\alpha 5(\mathrm{IV})^{38}$ were challenged with $\mathrm{CCl}_{4}$ (Fig. 4E-H, S8). Significantly less severe liver fibrosis developed in the $\mathrm{KO}$ mice after 4-week $\mathrm{CCl}_{4}$ treatment (Fig. 4E-I). $\alpha 5$ (IV) is expressed in the endothelial cells ${ }^{38} . \alpha 5(\mathrm{IV})$ partially colocalized with endothelial cell marker CD31 in the liver (Fig. 5A). Portal angiogenesis was reported to attenuate fibrogenesis, whereas sinusoid capillarization was believed to promote fibrogenesis $^{39}$. Despite knockdown of $\alpha 5$ (IV) impaired endothelial cell proliferation in vitro (Fig. S8A and S8B) ${ }^{38}, \alpha 5$ (IV) deficiency did not affect either portal angiogenesis or sinusoid capillarization (Fig. S8C-S8F).

\section{Minor type IV collagens regulate hepatic stellate cell activation and hepatocyte proliferation}

Hepatic stellate cells (HSCs) are the main producers of ECM in hepatocytic damage-induced liver fibrosis ${ }^{40,41}$. After 4-week $\mathrm{CCl}_{4}$ treatment, $\alpha 5$ (IV) partially colocalized with the HSC marker PDGFR $\alpha$ (Fig. 5A). At the fibrotic lesions, $\alpha 5(\mathrm{IV})$ was expressed in majority of the $\alpha \mathrm{SMA}$ and type I collagen (Col I)-positive activated HSCs (Fig. 5A). Knockdown of $\alpha 5$ (IV) in CSFC-8B HSC cells significantly reduced basal and TGF- $\beta$-induced expression of type I collagen and $\alpha$ SMA (Fig. $5 \mathrm{~B}$ ). $\alpha 5$ (IV) deficiency in lung cancer cells resulted in decreased expression of non-integrin collagen receptor discoidin domain receptor-1 (DDR1), and $\alpha 5$ (IV) regulates cell proliferation via DDR $1^{38}$. DDR1 is mainly expressed in epithelial cells, and its family member DDR2 is expressed in mesenchymal cells ${ }^{42}$. While DDR1 is not expressed in HSCs, DDR2 expression is induced in HSCs during liver injury ${ }^{43}$. DDR2 is induced in activated HSCs and is required for HSC activation. TGF- $\beta$ treatment increased DDR2 expression in CSFC-8B cells (Fig. 5B). DDR2 knockdown reduced expression of type I collagen and $\alpha \mathrm{SMA}$ in CSFC-8B cells (Fig. S9A). DDR2 expression was significantly reduced in $\alpha 5(I V)$-deficient livers (Fig. S9B and S9C). Knockdown of $\alpha 5(\mathrm{IV})$ in CSFC-8B cells significantly reduced DDR2 expression (Fig. $5 \mathrm{~B}$ ), suggesting $\alpha 5$ (IV) may regulate HSC activation and liver fibrosis through DDR2.

Damage-induced liver fibrosis is a consequence of imbalanced tissue damage and tissue repair. $\alpha 5$ (IV) deficiency may promote functional liver regeneration upon $\mathrm{CCl}_{4}$ challenge. $\alpha 5$ (IV) deficiency did not affect hepatocyte cell death after 4-week $\mathrm{CCl}_{4}$ treatment (Figs. 5C, D). However, $\alpha 5$ (IV) deficiency significantly increased the numbers of Ki67-positive proliferating cells after 4-week $\mathrm{CCl}_{4}$ treatment (Figs. 5C, D). Consistently, increased PCNA and cyclin D1 expression was detected in $\alpha 5$ (IV)deficient livers after 4-week $\mathrm{CCl}_{4}$ treatment (Fig. S10A and S10B). Upon injury, hepatocytes proliferate to recover from damage. Significantly more proliferating HNF4 $\alpha$ positive hepatocytes were evident in $\alpha 5($ IV)-deficient livers after 4-week $\mathrm{CCl}_{4}$ treatment (Figs. 5E, F). Activation of ERK1/2 and AKT signaling pathways play a major role in liver regeneration ${ }^{44,45}$. Consistent to higher percentage of proliferating hepatocytes, phosphorylation of ERK1/2 and AKT was significantly increased in $\alpha 5(\mathrm{IV})$-deficient livers after 4-week $\mathrm{CCl}_{4}$ treatment (Fig. S10C and S10D).

\section{Discussion}

Liver cirrhosis remains major health problem ${ }^{1,2}$. With the progress on diagnosis of asymptomatic early-stage liver cirrhosis, prognostic biomarkers are becoming even more urgent medical needs to identify cirrhotic patients at high risk developing advanced stage disease, and to guide the follow-up therapy after diagnosis. In this study, we analyzed dynamic changes of hepatic matrisome in the development of liver fibrosis, and identified matrisomal protein signatures as potential prognostic biomarkers for early-stage liver cirrhosis, in which minor type IV collagens play prominent roles in the development of liver fibrosis.

Serum biomarkers are currently available for non-invasive evaluation of liver fibrosis in chronic liver disease, e. g. Fibrotest@, AST to Platelet Ratio (APRI), FibroSpectII@, Enhanced Liver Fibrosis score ${ }^{\circledR}(E L F)^{3}$. Liver stiffness measurement with transient elastography and other liver 


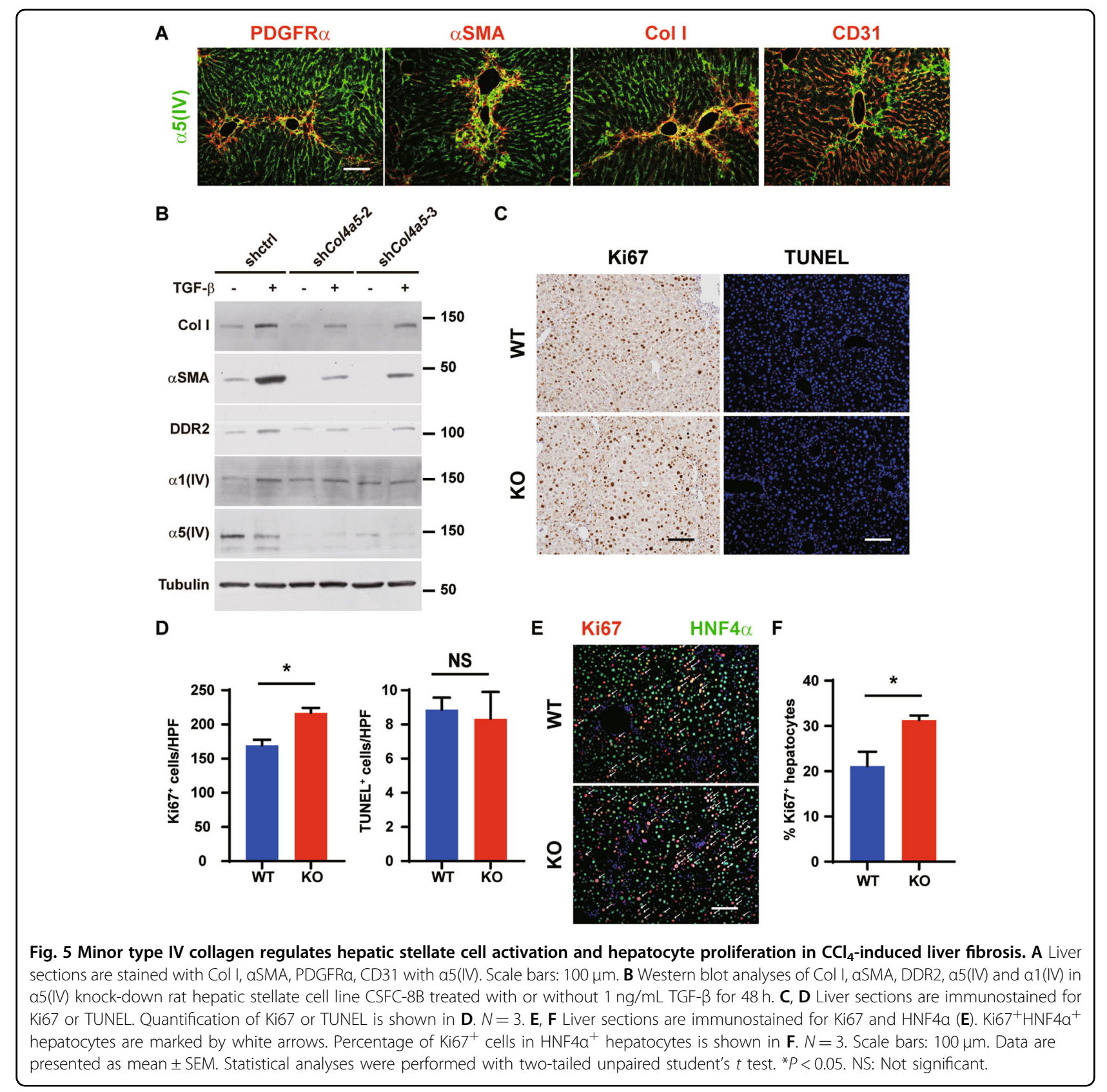

elasticity-based imaging techniques offer another noninvasive approach for early diagnosis of liver fibrosis and cirrhosis $^{3}$. Despite the success in diagnosis of liver cirrhosis, in particular asymptomatic early-stage cirrhosis, these diagnostic methods are not robust to classify early-stage cirrhosis patients into prognostic subgroups ${ }^{3}$. Age, sex, platelet count, and aspartate aminotransferase/alanine aminotransferase ratio were identified independently associated with mortality of chronic HCV infection and compensated advanced liver fibrosis ${ }^{46}$. Child-Pugh score, consisting of total bilirubin level, serum albumin level, prothrombin time, the degree of ascites, and the grade of hepatic encephalopathy, is used to assess the prognosis of liver cirrhosis ${ }^{4}$. In addition to clinical variables, gene signatures have been used to assess the clinical outcome of complex diseases. A prognostic 186-gene signature was reported to predict disease progression to advanced stage cirrhosis, patient survival and hepatocellular carcinoma development of HCV-related Child-Pugh Class A earlystage cirrhosis patients ${ }^{5,47}$.

Liver fibrosis is part of the general wound healing response to many causes of chronic injury ${ }^{7,8}$. Liver fibrosis and cirrhosis are characterized by aberrant deposition of ECM proteins. Individual ECM components have been 
proposed as diagnostic and prognostic biomarkers for liver fibrosis and cirrhosis. Matrisomal proteins are part of the 186-gene signature ${ }^{5,47}$, including collagens, glycoproteins, proteases, and secreted factors. Elastin is a key glycoprotein that provides elasticity to tissues. Although elastin is weakly expressed in normal liver, elastin is abundantly deposited at late stages of liver fibrosis ${ }^{48}$. Hepatic elastin content is predictive of adverse outcome in advanced fibrotic liver disease ${ }^{49}$. Elastin imaging enables staging and longitudinal monitoring of kidney fibrosis therapeutic interventions ${ }^{50}$. ECM remodeling is highly dynamic and complex in tissue homeostasis and diseases. Proteomic analyses of matrisome composition of normal and diseased tissues provide insights into mechanisms regulating fibrotic diseases ${ }^{10-17}$. In this study, we identified cluster- 3 and cluster- 4 matrisomal proteins as potential prognostic biomarkers for earlystage cirrhosis. Cluster-3 matrisomal proteins (24 genes) are responsible for fibrinolysis and homeostatic remodeling. Cluster-3 signature is associated with good prognosis of HCV-related early-stage cirrhosis patients (Fig. 2, Table S3 and S4). Cluster-4 ECM signature (52 genes) includes core ECM proteins previously reported to regulate liver fibrogenesis, e.g., collagens, elastin and small leucine-rich proteoglycans ${ }^{51}$. Cluster- 4 signature predicts poor prognosis of HCV-related early-stage cirrhosis patients (Fig. 2, Table S3 and S5). In particular, patients with low cluster-3 signature and high cluster-4 signature had significantly shorter disease progression-free survival and overall survival (Fig. 2), highlighting the importance of balanced tissue repair and fibrogenesis in the development of cirrhosis.

Besides cluster- 3 and cluster- 4 signatures, BM signature is potent in predicting early-stage liver cirrhosis disease progression and patient survival (Fig. 3). At the stage when fibrotic lesions were prominent, components of $\mathrm{BMs}^{33}$ were enriched in the hepatic matrix, including type IV, XV, XVIII collagens, laminin 521, nidogens, and heperan sulfate proteoglycans perlecan and agrin (Table S2). A 14-gene signature, present in both cluster- 4 and basement membrane signatures, is potent in predicting disease progression and patient survival (Fig. 3). Clinical variables, e. g. bilirubin level and platelet count, are used to assess the prognosis of liver cirrhosis ${ }^{4}$. Interestingly, patients with high basement membrane signature/14-gene signature and high bilirubin level had the shortest disease progression-free survival and overall survival, while patients with low basement membrane signature/14-gene signature and low bilirubin level had the best prognosis (Fig. S7).

Type IV collagens, core components of BMs, are deposited at the Disse space ${ }^{35}$ and upregulated in fibrotic livers ${ }^{34}$. Type IV collagen networks are essential in maintaining BM structure ${ }^{36,37}$. Major and minor type IV collagens were both extensively deposited to fibrotic lesions (Fig. 3F). Minor type IV collagen expression and structure were dynamically changed during the development of liver fibrosis (Fig. 4 and S7), suggesting minor type IV collagens may play prominent roles in regulating liver fibrosis. Minor type IV collagens are highly expressed in activated hepatic stellate cells. Deficiency of minor type IV collagens resulted in reduced activation of hepatic stellate cells and much reduced liver fibrosis (Figs. 4 and 5). On the other hand, minor type IV collagens regulated hepatocyte proliferation and the wound healing process (Fig. 5). The essential roles of type IV collagens in the development of liver fibrosis indicate that the prognostic value of basement membrane is closely related to its functions regulating liver cirrhosis development. Minor type IV collagens may regulate HSC activation via nonintegrin collagen receptor DDR2, which was previously reported to be induced in HSCs during liver injury and mediates HSC activation ${ }^{43}$. Type IV collagens bind soluble glycoproteins, proteoglycans and growth factors in a tissue-specific manner to establish fully functional $\mathrm{BMs}^{52}$. Hepatocyte growth factor (HGF) is an HSC-derived paracrine factor regulating liver cancer cell proliferation via its receptor c-Met and downstream MAPK and Akt pathways ${ }^{53}$. It warrants further investigation whether minor type IV collagens regulated hepatocyte proliferation through HGF and possibly other growth factors.

In summary, unique matrisomal proteins were identified for tissue damage/inflammation, transitional ECM remodeling and fibrogenesis. Such matrisomal protein signatures, in particular basement membrane signature, are predictive of early-stage cirrhosis progression and patient survival, and may help development of biomarkers for the prediction of clinical outcomes of liver cirrhosis patients at very early stage.

\section{Materials and methods \\ Liver injury and fibrosis models}

Male C57BL/6 mice were purchased from SLAC (Shanghai). Col4a5 knockout mice were maintained in C57/ BL6 background as described ${ }^{38}$. 8-week-old wild-type or Col4a 5 knockout mice were randomly divided to four groups, and were intraperitoneally injected with $1 \mu \mathrm{l} / \mathrm{g}$ body weight $\mathrm{CCl}_{4}$ (dissolved in olive oil at a ratio of 1:4) (SigmaAldrich) or olive oil twice a week for 1 day, 1 week or 4 weeks to induce liver injury and liver fibrosis as descri$\operatorname{bed}^{23}$. The mice were euthanized by inhaling $\mathrm{CO}_{2}$ at 1 day, 1 week and 4 weeks after initial $\mathrm{CCl}_{4}$ injection. Livers were fixed in $4 \%$ paraformaldehyde and processed for paraffin embedding, or were snap frozen in liquid nitrogen and stored at $-80^{\circ} \mathrm{C}$. No blinding was performed.

\section{Decellularization and ECM protein enrichment}

Three biological repeats were obtained for each represented stage. Decellularization and ECM protein 
enrichment were performed as described with modifications $^{54}$. $\sim 50 \mathrm{mg}$ liver was homogenated in $2 \mathrm{~mL}$ extraction buffer $(10 \mathrm{mM}$ Tris- $\mathrm{HCl}, \mathrm{pH} 7.5,150 \mathrm{mM} \mathrm{NaCl}, 1 \%$ Triton X-100, $10 \mathrm{mM}$ EDTA, $1 \times$ Roche protease inhibitors), and incubated for $16 \mathrm{~h}$ to solubilize cellular proteins. Pellets from centrifuged tissue homogenates were resuspended in extraction buffer and incubated for another $16 \mathrm{~h}$. Samples were then centrifuged and the remaining pellet was washed once with $10 \mathrm{mM}$ Tris- $\mathrm{HCl}, \mathrm{pH} 7.5$, $150 \mathrm{mM} \mathrm{NaCl}$, and incubated overnight in deoxyribonuclease buffer $(100 \mathrm{ug} / \mathrm{ml}$ deoxyribonuclease I in $10 \mathrm{mM}$ Tris- $\mathrm{HCl}, \mathrm{pH} 7.5,2.5 \mathrm{mM} \mathrm{MgCl} 2,0.5 \mathrm{mM} \mathrm{CaCl}_{2}$ ) to degrade DNA. The samples were centrifuged, and the final pellet was washed with PBS three times to yield the enriched ECM fraction. All steps were carried out at $4{ }^{\circ} \mathrm{C}$ to minimize proteolysis.

\section{LC-MS/MS for ECM proteome analysis}

Enriched ECM proteins were digested into peptides as previously reported ${ }^{55}$ with slight modification. In brief, $\sim 5 \mathrm{mg}$ dry weight ECM-enriched pellets were resuspended in $8 \mathrm{M}$ urea with a final concentration of $40 \mathrm{mM}$ DTT at $1400 \mathrm{rpm}$ for $2 \mathrm{~h}$ at $37^{\circ} \mathrm{C}$. Protein samples were cooled to room temperature and IAA was added to a final concentration of $100 \mathrm{mM}$. Akylation reactions last $30 \mathrm{~min}$ in darkness at room temperature (RT). Urea was diluted to $2 \mathrm{M}$ with $100 \mathrm{mM}$ ammonium bicarbonate, $\mathrm{pH}$ 8.0. Deglycosylation with $1000 \mathrm{U}$ PNGase F per sample was preceded for $2 \mathrm{~h}$ at $37^{\circ} \mathrm{C}$ with continuous agitation at $1400 \mathrm{rpm} .1 \mu \mathrm{g}$ endoproteinase Lys- $\mathrm{C}$ was then added to each sample and incubated at $1400 \mathrm{rpm}$ for $2 \mathrm{~h}$ at $37^{\circ} \mathrm{C}$. The first aliquot of $3 \mu \mathrm{g}$ trypsin was added to each sample, followed by overnight incubation at $37^{\circ} \mathrm{C}$ with continuous agitation at $1400 \mathrm{rpm}$. A second aliquot of $3 \mu \mathrm{g}$ trypsin was then added to each sample and incubated at $1400 \mathrm{rpm}$ for an additional $2 \mathrm{~h}$ at $37^{\circ} \mathrm{C}$. $\sim 1 \mu \mathrm{l}$ freshly prepared $50 \%$ TFA per sample was added to inactivate trypsin after the protein digestion. After centrifugation at $16,000 \times g$ for $5 \mathrm{~min}$ at RT, all the supernatants were collected into clean tubes, desalted by StageTips $^{56}$. Peptide concentrations were measured assuming a mean tryptophan content of $1.3 \%{ }^{57}$.

The purified peptide samples were analyzed on the Thermo Scientific ${ }^{\mathrm{TM}}$ Q Exactive ${ }^{\mathrm{TM}}$ HF-X hybrid quadrupoleOrbitrap mass spectrometer coupled to HPLC via a nanoelectrospray ion source. The LC-MS/MS method was similar to the previous reports ${ }^{58,59}$ with minor modifications. Briefly, each peptide sample was loaded directly onto a spray analytical column (75 $\mu \mathrm{m}$ inner diameter, $~ 150 \mathrm{~mm}$ length) packed in-house with ReproSil-Pur C18-AQ, $3 \mu \mathrm{m}$ resin (Dr. Maisch, $\mathrm{GmbH})$ using a 60 min gradient B $(0.1 \%$ formic acid in acetonitrile) from 5 to $90 \%$ at a flow rate of approximate $300 \mathrm{~nL} / \mathrm{min}$ after split. The analytical column was previously equilibrated to $95 \%$ Mobile Phase A $(0.1 \%$ formic acid in water) and $5 \%$ Mobile Phase B $(0.1 \%$ formic acid in acetonitrile) and maintained at a constant column flow of $300 \mathrm{~nL} / \mathrm{min}$. The gradient profile (min: \%B) was as shown in the following: 0:5, 2:8, 42:23, 50:40, 52:90, 60:90. A lock-mass $\mathrm{m} / \mathrm{z} 445.12003$ was used for internal calibration. Electrospray voltage $(2.5 \mathrm{kV})$ was applied between the analytical column and electrospray emitter. The capillary temperature was set at $300^{\circ} \mathrm{C}$. Orbitrap precursor spectra were collected from $300-1500 \mathrm{~m} / \mathrm{z}$ for $60 \mathrm{~min}$ at a resolution of $120 \mathrm{~K}$ (AGC target $3 \times 10^{6}$, maximum ion time of $20 \mathrm{msec}$ ) along with the top 25 data dependent Orbitrap HCD MS/MS spectra at a resolution of $15 \mathrm{~K}$ (AGC target $1 \times 10^{5}$, maximum ion time of $25 \mathrm{msec}$ ). Masses selected for MS/MS were isolated at a width of $1.2 \mathrm{~m} / \mathrm{z}$ and fragmented using a normalized collision energy of $28 \%$. Exclude isotopes was set to 'on', and charge state screening was enabled to reject unassigned, $1+, 7+, 8+$, and $>8+$ ions with a dynamic exclusion time of $30 \mathrm{~s}$ to discriminate against previously analyzed ions between $\pm 12 \mathrm{ppm}$.

\section{Quantification of ECM proteomics data, protein annotation and functional analysis}

Considering various intracellular and extracellular posttranslational modifications (PTMs) involved in the generation and maturation of ECM proteins (especially for collagens) $^{60,61}$, dominant PTMs were firstly scanned in the MS data using an open search engine pFind (V3.1.5, 64 bit for windows, released on Jan. 24, 2019) ${ }^{62}$. Carbamidomethyl $(\mathrm{C})$, oxidation $(\mathrm{M})$, deamidated $(\mathrm{N})$, hydroxyproline, and hydroxylysine were ranked the 1st, 2nd, 3rd, 8th, and 28th place with $9.56 \%, 6.62 \%, 5.60 \%, 0.88 \%$ and $0.09 \%$ of all identified spectra, respectively. All acquired spectra were then searched by MaxQuant version 1.6.2.10 against the UniProt Mouse protein database (07/2017 download, 82051 sequences) with contaminant sequences and decoy sequences for label-free peptide quantification ${ }^{63,64}$. Main search parameters used (Carbamidomethyl (C), oxidation $(\mathrm{M})$ and deamidated $(\mathrm{N})$ ) were specified as variable modifications. The precursor mass tolerance for protein identification on MS was $4.5 \mathrm{ppm}$, and the product ion tolerance for MS/MS was $7 \mathrm{ppm}$. Full cleavage by trypsin was used, with up to two missed cleavages allowed. A $1 \%$ FDR at the PSM, peptide and protein level, peptides with a minimum length of seven amino acids. Label-free quantification was performed with normalized protein intensity using a minimum ratio count of 2 . As for proteins with hydroxyproline and hydroxylysine, carbamidomethyl (C), oxidation (M), hydroxyproline and hydroxylysine were specified as variable modifications. Other parameters were the same as the above description and removed all the scans identified in previous searching by manual check. Protein classification was performed with in silico Matrisome annotation tool (mouse Matrisome) ${ }^{10}$. $\mathrm{R}$ version 3.6.0 (http://www.R-project.org/) was used to perform massspec data normalization, statistical analysis, PCA (principal 
component analysis), HCA (hierarchical cluster analysis), and C-Means Clustering. After averaging the three biological replicates for each time points, the data matrix was standardized using $\mathrm{R}$ package Mfuzz. 152 matrisomal proteins were classified into 4 clusters with the degree of fuzzification $m=2$ based on fuzzy c-means (FCM) algorithm. This parameter combination gave a clear temporal classification for the four time points, without overlapping clusters or empty clusters. The maximum membership score of the given protein was considered for partitioning it to the corresponding cluster (Table S2). Kruskal-Willis test was used to select differentially expressed proteins. Gene ontology (GO) analysis was performed with the DAVID online tool. Top GO categories were selected according to the $P$ value after Benjamini-Hochberg correction. Gene set enrichment analyses (GSEA) were performed on matrisome gene signatures ${ }^{10}$ obtained from the MSigDB database v5.0 (March 2015 release) ${ }^{27}$, or from the clusters identified in the mass-spec analysis in this study. Gene signatures used in the GSEA analysis are provided in Table S8. Statistical significance of GSEA analysis was assessed by comparing the enrichment score to enrichment results generated from 1,000 random permutations of the gene set to obtain $P$ values (nominal $P$ value).

\section{Cell lines}

CSFC-8B hepatic stellate cells (ATCC) were maintained in RPMI 1640 (Hyclone) supplemented with 10\% FBS (Biochrom). 293T and EA.hy926 cells (ATCC) were cultured in DMEM (GIBCO) with 10\% FBS. All cell lines were routinely tested for mycoplasma contamination. shRNAs targeting Col4a5 were cloned into pLKO.1-puro lenti-viral vector (Addgene). The target sequences are: 5'-CAACAAGA TGAAGAGCACCAAC-3' (shScram), 5'-GGGTGATGAT GGAATTCCA-3' (shCOL4A5-10), 5'-GCCAGAGCAAA GTCTCTATTA-3' (shCOL4A5-16), 5'-GCTCCTGTTTG GAAGAATTTC-3' (Rat shCol4a5-2), 5'-GCAATGGA CTCCCAGGCTTTG-3' (Rat shCol4a5-3). After viral infection, cells were selected with puromycin to generate stable cell lines. At least two batches of stable cell lines were generated for each experiment. Experiments were performed in triplicates and repeated at least twice. Cell Counting Kit-8 (CCK8) and 5-ethynyl-2'-deoxyuridine (EdU) incorporation assays were performed according to manufacturer's protocol.

\section{Histological and immunohistochemical staining}

Paraffin-embedded liver tissues were sectioned and stained with hematoxylin and eosin (HE) or Picrosirius Red (Sigma) for gross histology and fibrosis assessment. The immunohistochemical staining was performed using Avidin-Biotin Complex (ABC) method as previously described $^{65}$. Briefly, deparaffined and rehydrated $5 \mu \mathrm{m}$ liver sections were subjected to heat-induced epitope retrieval with $10 \mathrm{mM}$ Citrate at $\mathrm{pH} 2.0$ (for $\alpha 2$ (IV) and $\alpha 5$ (IV)) or at $\mathrm{pH}$ 6.0. Sections were incubated with diluted primary antibodies at $4{ }^{\circ} \mathrm{C}$ overnight, followed by incubation with biotinylated secondary antibodies and horseradish peroxidase-conjugated $\mathrm{ABC}$ complex. The primary antibodies used are listed in Table S9. Signals were developed with $\mathrm{DAB}$ chromogen and counterstained with hematoxylin. For multiplex immunofluorescent staining, $\mathrm{Opal}^{\mathrm{TM}}$ fluorophores (PerkinElmer) were used to visualized the signal. Primary and secondary antibodies in previous round immunostaining were stripped by microwave treatment before staining with a second primary antibody. Sections were treated with TrueBlack ${ }^{\circledR}$ Lipofuscin Autofluorescence Quencher (Biotium) before DAPI counterstaining and mounting. Sections were viewed under microscope BX53 with an UPlanSAPO $\times 20$ objective/0.75 (OLYMPUS, Inc.). Images were captured with a digital camera (IX-SPT; OLYMPUS, Inc.) and Digital Acquire software (DPController; OLYMPUS, Inc.). Automated scoring and quantitative evaluation of $\mathrm{DAB}$ stained IHC images are performed using open source IHC Profiler Plugin ${ }^{66}$ in ImageJ software. H-score was calculated by adding the percentage of high positive $(\times 3)$, positive $(\times 2)$, and low positive cells $(\times 1)$ as described ${ }^{67}$. Hscore has a possible range of $0-300$. Quantification of picrosirius red-positive area, integrated fluorescent density, and numbers of Ki67-positive or TUNEL-positive cells are performed using ImageJ software. Percentage of Ki67-positive cells in HNF4a-positive hepatocytes are analyzed with InForm ${ }^{\circledR}$ software (PerkinElmer). The number of CD31-positive vessels surrounding the portal area and the CD31-positive capillarization of liver sinusoids were manually counted as described ${ }^{39}$. All quantification was performed on pictures taken from at least 5 randomly selected $20 \times$ fields per mouse. Three to four mice per treatment group were analyzed.

\section{Western blot and quantitative RT-PCR analysis}

Livers were homogenated in SDS sample buffer or Trizol reagents (Invitrogen). Western blot and quantitative RT-PCR analyses were performed as previously described ${ }^{68}$. The primary antibodies and primers used are listed in Tables S9 and S10, respectively. Gene expression levels were normalized to Actin. All experiments were performed at least twice.

\section{Early-stage liver cirrhosis patient disease progression and survival analyses}

Previously published Child-Pugh Class A early-stage liver cirrhosis microarray data (GSE15654) $)^{5}$ is used to assess the prognostic potential of matrisome cluster signatures. To avoid potential heterogeneity among the samples, 216 samples were randomly split into two data sets with similar size (107 and 109 cases). The two cohorts 
have similar clinicopathologic characteristics as assessed by chi-square test (Table S4). Gene set enrichment scores of each ECM signature were calculated for each sample using Gene Set Variation Analysis (GSVA) ${ }^{69}$. Survival analysis was performed using the uni-variable and multivariable Cox regression and Kaplan-Meier (Log-Rank test) method to evaluate the correlation of the ECM signature GSVA score with disease progression and overall survival in these two data sets.

\section{Statistical analysis}

Variance similarity between the groups is being statistically compared. Data meet the assumptions of the tests. No data were excluded from the analysis. Sample size was based on previous research experience. Sample numbers were indicated in figure legends. Statistical significance between conditions was assessed by unpaired two-tailed Student's $t$-tests or one-way Anova followed by Tukey's post-hoc analysis using GraphPad Prism. All error bars represent SEM.

\section{Acknowledgements}

The authors acknowledge the Genome Tagging Project (GTP) Center, Center for Excellence in Molecular Cell Science, Chinese Academy of Sciences, and Drs. Baojin Wu and Guoyuan Chen for technical support.

\section{Funding}

The work was supported by the National Natural Science Foundation of China (81430067, 32070789), Ministry of Science and Technology (2020YFA0803203, 2017YFA0505502), the Strategic Priority Research Program of the Chinese Academy of Sciences (XDA12010100), the National Ten Thousand Talents Program and the SA-SIBS Scholarship program.

\begin{abstract}
Author details
${ }^{1}$ State Key Laboratory of Cell Biology, Shanghai Institute of Biochemistry and Cell Biology, Center for Excellence in Molecular Cell Science, Chinese Academy of Sciences, 200031 Shanghai, China. ${ }^{2}$ University of Chinese Academy of Sciences, 100049 Beijing, China. ${ }^{3}$ CAS Key Laboratory of Systems Biology, Shanghai Institute of Biochemistry and Cell Biology, Center for Excellence in Molecular Cell Science, Chinese Academy of Sciences, 200031 Shanghai, China. ${ }^{4}$ School of Life Science, Hangzhou Institute for Advanced Study, University of Chinese Academy of Sciences, 310024 Hangzhou, China. ${ }^{5}$ Center for Single-Cell Omics, School of Public Health, Shanghai Jiao Tong University School of Medicine, 200025 Shanghai, China. ${ }^{6}$ CAS Key Laboratory of Systems Biology, Hangzhou Institute for Advanced Study, University of Chinese Academy of Sciences, 310024 Hangzhou, China. ${ }^{7}$ School of Life Science and Technology, Shanghai Tech University, 201210 Shanghai, China
\end{abstract}

\section{Author contributions}

Y.W., C.L., Y.C.-study design; acquisition, analysis, and interpretation of data; assistance in manuscript preparation. K.X., Y.Z., Y.Q., Y.W.-acquisition of data; J.C.-interpretation of data; assistance in manuscript preparation. R.Z., G.G.-study concept and design, interpretation of data, study supervision, manuscript preparation.

\section{Conflict of interest}

The authors declare that they have no conflict of interest.

\section{Ethics}

All mice were housed in a specific pathogen-free environment at the Shanghai Institute of Biochemistry and Cell Biology (SIBCB) and treated in strict accordance with protocols approved by the Institutional Animal Care and Use Committee of SIBCB (Approval number: SIBCB-NAF-15-003-S325-006).

\section{Publisher's note}

Springer Nature remains neutral with regard to jurisdictional claims in published maps and institutional affiliations.

\section{Supplementary information}

The online version contains supplementary material available at https://doi. org/10.1038/s41419-021-03443-y.

Received: 1 November 2020 Revised: 12 January 2021 Accepted: 15 January 2021

Published online: 08 February 2021

\section{References}

1. Bataller, R. \& Brenner, D. A. Liver fibrosis. J. Clin. Invest. 115, 209-218 (2005).

2. Schuppan, D. \& Afdhal, N. H. Liver cirrhosis. Lancet 371, 838-851 (2008).

3. European Association for Study of the Liver \& Asociacion Latinoamericana para el Estudio del Higado EASL-ALEH clinical practice guidelines: non-invasive tests for evaluation of liver disease severity and prognosis. J. Hepatol. 63, 237-264 (2015).

4. Pugh, R. N., Murray-Lyon, I. M., Dawson, J. L., Pietroni, M. C. \& Williams, R. Transection of the oesophagus for bleeding oesophageal varices. Br. J. Surg. 60, 646-649 (1973).

5. Hoshida, Y. et al. Prognostic gene expression signature for patients with hepatitis C-related early-stage cirrhosis. Gastroenterology 144, 1024-1030 (2013).

6. Zhang, D. Y. et al. A hepatic stellate cell gene expression signature associated with outcomes in hepatitis C cirrhosis and hepatocellular carcinoma after curative resection. Gut 65, 1754-1764 (2016).

7. Pellicoro, A., Ramachandran, P., Iredale, J. P. \& Fallowfield, J. A. Liver fibrosis and repair: immune regulation of wound healing in a solid organ. Nat. Rev. Immunol. 14, 181-194 (2014).

8. Iredale, J. P. Models of liver fibrosis: exploring the dynamic nature of inflammation and repair in a solid organ. J. Clin. Invest. 117, 539-548 (2007).

9. Hynes, R. O. \& Naba, A. Overview of the matrisome-an inventory of extracellular matrix constituents and functions. Cold Spring Harb. Perspect. Biol. 4, a004903 (2012).

10. Naba, A. et al. The matrisome: in silico definition and in vivo characterization by proteomics of normal and tumor extracellular matrices. Mol. Cell Proteom. 11, M111 014647 (2012).

11. Bergmeier, V. et al. Identification of a myofibroblast-specific expression signature in skin wounds. Matrix Biol. 65, 59-74 (2018).

12. Massey, V. L. et al. The hepatic "matrisome" responds dynamically to injury: characterization of transitional changes to the extracellular matrix in mice. Hepatology 65, 969-982 (2017).

13. Naba, A. et al. Characterization of the extracellular matrix of normal and diseased tissues using proteomics. J. Proteome Res. 16, 3083-3091 (2017).

14. Yuzhalin, A. E., Urbonas, T., Silva, M. A., Muschel, R. J. \& Gordon-Weeks, A. N. A core matrisome gene signature predicts cancer outcome. Br. J. Cancer 118, 435-440 (2018).

15. Gocheva, $V$. et al. Quantitative proteomics identify Tenascin- $C$ as a promoter of lung cancer progression and contributor to a signature prognostic of patient survival. Proc. Natl Acad. Sci. USA 114, E5625-E5634 (2017).

16. Lai, K. K. et al. Extracellular matrix dynamics in hepatocarcinogenesis: a comparative proteomics study of PDGFC transgenic and Pten null mouse models. PLoS Genet. 7, e1002147 (2011).

17. Schiller, H. B. et al. Time- and compartment-resolved proteome profiling of the extracellular niche in lung injury and repair. Mol. Syst. Biol. 11, 819 (2015).

18. Martin, D. R. et al. Proteomics identifies a convergent innate response to infective endocarditis and extensive proteolysis in vegetation components. JCl Insight 5, e135317 (2020).

19. Odenthal, M., Neubauer, K., Meyer zum Buschenfelde, K. H. \& Ramadori, G. Localization and mRNA steady-state level of cellular fibronectin in rat liver undergoing a CCl4-induced acute damage or fibrosis. Biochim. Biophys. Acta 1181, 266-272 (1993).

20. Gillis, S. E. \& Nagy, L. E. Deposition of cellular fibronectin increases before stellate cell activation in rat liver during ethanol feeding. Alcohol Clin. Exp. Res. 21, 857-861 (1997)

21. Altrock, E. et al. Inhibition of fibronectin deposition improves experimental liver fibrosis. J. Hepatol. 62, 625-633 (2015). 
22. Delire, B., Starkel, P. \& Leclercq, I. Animal models for fibrotic liver diseases: what we have, what we need, and what is under development. J. Clin. Transl. Hepatol. 3, 53-66 (2015).

23. Kim, Y. O., Popov, Y. \& Schuppan, D. Optimized mouse models for liver fibrosis. Methods Mol. Biol. 1559, 279-296 (2017).

24. Krasny, L. et al. Comparative proteomic assessment of matrisome enrichment methodologies. Biochem J. 473, 3979-3995 (2016).

25. Ricard-Blum, S. The collagen family. Cold Spring Harb. Perspect. Biol. 3, a004978 (2011).

26. Qi, Y. \& Xu, R. Roles of PLODs in collagen synthesis and cancer progression. Front. Cell Dev. Biol. 6, 66 (2018).

27. Subramanian, A. et al. Gene set enrichment analysis: a knowledge-based approach for interpreting genome-wide expression profiles. Proc. Natl Acad. Sci. USA 102, 15545-15550 (2005).

28. Wang, S. et al. S100A8/A9 in inflammation. Front. Immunol. 9, 1298 (2018).

29. Huang, Y. et al. Matricellular protein periostin contributes to hepatic inflammation and fibrosis. Am. J. Pathol. 185, 786-797 (2015).

30. Marchant, J. K., Hahn, R. A., Linsenmayer, T. F. \& Birk, D. E. Reduction of type V collagen using a dominant-negative strategy alters the regulation of fibrillogenesis and results in the loss of corneal-specific fibril morphology. J. Cell Biol. 135, 1415-1426 (1996).

31. Kalluri, R. Basement membranes: structure, assembly and role in tumour angiogenesis. Nat. Rev. Cancer 3, 422-433 (2003).

32. Pozzi, A., Yurchenco, P. D. \& lozzo, R. V. The nature and biology of basement membranes. Matrix Biol. 57-58, 1-11 (2017).

33. Randles, M. J., Humphries, M. J. \& Lennon, R. Proteomic definitions of basement membrane composition in health and disease. Matrix Biol. 57-58, 12-28 (2017).

34. Mak, K. M. \& Mei, R. Basement membrane type IV collagen and laminin: an overview of their biology and value as fibrosis biomarkers of liver disease. Anat. Rec. 300, 1371-1390 (2017).

35. Martinez-Hernandez, A. The hepatic extracellular matrix. II. Electron immunohistochemical studies in rats with CCl4-induced cirrhosis. Lab. Invest. $\mathbf{5 3}$ 166-186 (1985).

36. Poschl, E. et al. Collagen IV is essential for basement membrane stability but dispensable for initiation of its assembly during early development. Development 131, 1619-1628 (2004).

37. Wu, Y. \& Ge, G. Complexity of type IV collagens: from network assembly to function. Biol. Chem. 400, 565-574 (2019).

38. Xiao, Q. et al. Minor type IV collagen alpha5 chain promotes cancer progression through discoidin domain receptor-1. PLoS Genet. 11, e1005249 (2015).

39. Xu, M. et al. LECT2, a ligand for Tie1, plays a crucial role in liver fibrogenesis. Cell 178, 1478-1492 e1420 (2019).

40. Friedman, S. L. Hepatic stellate cells: protean, multifunctional, and enigmatic cells of the liver. Physiol. Rev. 88, 125-172 (2008).

41. Yin, C., Evason, K. J., Asahina, K. \& Stainier, D. Y. Hepatic stellate cells in liver development, regeneration, and cancer. J. Clin. Invest 123, 1902-1910 (2013).

42. Fu, H. L. et al. Discoidin domain receptors: unique receptor tyrosine kinases in collagen-mediated signaling. J. Biol. Chem. 288, 7430-7437 (2013).

43. Olaso, E. et al. DDR2 receptor promotes MMP-2-mediated proliferation and invasion by hepatic stellate cells. J. Clin. Invest. 108, 1369-1378 (2001).

44. Morales-Ruiz, M., Santel, A., Ribera, J. \& Jimenez, W. The role of Akt in chronic liver disease and liver regeneration. Semin Liver Dis. 37, 11-16 (2017).

45. Kitade, M. et al. Specific fate decisions in adult hepatic progenitor cells driven by MET and EGFR signaling. Genes Dev. 27, 1706-1717 (2013).

46. van der Meer, A. J. et al. Reliable prediction of clinical outcome in patients with chronic HCV infection and compensated advanced hepatic fibrosis: a validated model using objective and readily available clinical parameters. Gut $\mathbf{6 4}$, 322-331 (2015).
47. King, L. Y. et al. A genomic and clinical prognostic index for hepatitis C-related early-stage cirrhosis that predicts clinical deterioration. Gut 64, 1296-1302 (2015).

48. Chen, W. et al. Dynamics of elastin in liver fibrosis: accumulates late during progression and degrades slowly in regression. J. Cell Physiol. 234, 22613-22622 (2019)

49. Kendall, T. J. et al. Hepatic elastin content is predictive of adverse outcome in advanced fibrotic liver disease. Histopathology 73, 90-100 (2018).

50. Sun, Q. et al. Elastin imaging enables noninvasive staging and treatment monitoring of kidney fibrosis. Sci. Transl. Med. 11, eaat4865 (2019).

51. Krishnan, A. et al. Lumican, an extracellular matrix proteoglycan, is a novel requisite for hepatic fibrosis. Lab. Invest. 92, 1712-1725 (2012).

52. Mayorca-Guiliani, A. E. et al. ISDoT: in situ decellularization of tissues for highresolution imaging and proteomic analysis of native extracellular matrix. Nat. Med. 23, 890-898 (2017)

53. Lau, E. Y. et al. Cancer-associated fibroblasts regulate tumor-initiating cell plasticity in hepatocellular carcinoma through c-Met/FRA1/HEY1 Signaling. Cell Rep. 15, 1175-1189 (2016)

54. Lennon, R. et al. Global analysis reveals the complexity of the human glomerular extracellular matrix. J. Am. Soc. Nephrol. 25, 939-951 (2014).

55. Naba A., Clauser K. R. \& Hynes R. O. Enrichment of extracellular matrix proteins from tissues and digestion into peptides for mass spectrometry analysis. J. Vis. Exp. e53057 (2015)

56. Rappsilber, J., Mann, M. \& Ishihama, Y. Protocol for micro-purification, enrichment, pre-fractionation and storage of peptides for proteomics using StageTips. Nat. Protoc. 2, 1896-1906 (2007).

57. Nielsen, P. A. et al. Proteomic mapping of brain plasma membrane proteins. Mol. Cell Proteom. 4, 402-408 (2005).

58. Li, M. et al. Dysfunction of PLA2G6 and CYP2C44-associated network signals imminent carcinogenesis from chronic inflammation to hepatocellular carcinoma. J. Mol. Cell Biol. 9, 489-503 (2017).

59. Tang, Y. et al. RPS3A positively regulates the mitochondrial function of human periaortic adipose tissue and is associated with coronary artery diseases. Cell Discov. 4, 52 (2018).

60. Ishikawa, Y. \& Bachinger, H. P. A molecular ensemble in the rER for procollagen maturation. Biochim. Biophys. Acta 1833, 2479-2491 (2013).

61. Basak, T. et al. Comprehensive characterization of glycosylation and hydroxylation of basement membrane collagen IV by high-resolution mass spectrometry. J. Proteome Res. 15, 245-258 (2016).

62. Chi, $\mathrm{H}$. et al. Comprehensive identification of peptides in tandem mass spectra using an efficient open search engine. Nat. Biotechnol. 36, 1059-1061 (2018).

63. Cox, J. \& Mann, M. MaxQuant enables high peptide identification rates, individualized p.p.b.-range mass accuracies and proteome-wide protein quantification. Nat. Biotechnol. 26, 1367-1372 (2008).

64. Cox, J. et al. Accurate proteome-wide label-free quantification by delayed normalization and maximal peptide ratio extraction, termed MaxLFQ. Mol. Cell Proteom. 13, 2513-2526 (2014).

65. Zhang, R. et al. FSP1-positive fibroblasts are adipogenic niche and regulate adipose homeostasis. PLoS Biol. 16, e2001493 (2018).

66. Varghese, F., Bukhari, A. B., Malhotra, R. \& De, A. IHC Profiler: an open source plugin for the quantitative evaluation and automated scoring of immunohistochemistry images of human tissue samples. PLOS ONE 9, e96801 (2014).

67. Zhang, R. et al. Th-POK regulates mammary gland lactation through mTORSREBP pathway. PLoS Genet. 14, e1007211 (2018).

68. Gao, Y. et al. LKB1 inhibits lung cancer progression through lysyl oxidase and extracellular matrix remodeling. Proc. Natl Acad. Sci. USA 107, 18892-18897 (2010).

69. Hanzelmann, S., Castelo, R. \& Guinney, J. GSVA: gene set variation analysis for microarray and RNA-seq data. BMC Bioinform. 14, 7 (2013). 